\title{
Situación de la seguridad social de los trabajadores asalariados de 50 a 59 años en el Estado de México, 2015
}

\author{
Sergio Cuauhtémoc Gaxiola Robles Linares, Daniel Lozano \\ Keymolen, Bernardino Jaciel Montoya Arce y Yuliana \\ Gabriela Román Sánchez
}

Universidad Autónoma del Estado de México

\section{Resumen}

El objetivo del artículo consiste en analizar la relación de la seguridad social con las características sociodemográficas y laborales de los trabajadores asalariados de 50 a 59 años, en el Estado de México. Para esta investigación se utilizó la técnica estadística de Análisis de Correspondencia Múltiple, con la finalidad de mostrar gráficamente las relaciones entre la seguridad social y las características sociodemográficas de la población de estudio. La fuente de información que se utilizó fue la Encuesta Intercensal 2015. Uno de los resultados expone que la población analizada se agrupa en tres conjuntos, diferenciados por grados de vulnerabilidad, y que presentan componentes de fenómenos sociales como la desigualdad y la heterogeneidad en el mercado laboral.

Palabras clave: Seguridad social, pensiones, Estado de México, envejecimiento, análisis de correspondencia múltiple.

\section{Abstract}

The objective of this article is to analyze the relationship between social security and the sociodemographic and labor characteristics of salaried workers from 50 to 59 years old, in the State of Mexico. For this research the statistical technique of Multiple Correspondence Analysis was used with the purpose of graphically showing the relationships between social security and the sociodemographic characteristics of the studied population. The source of information used was the Intercensal Survey 2015. One of the results shows that in the analyzed population three groups were formed, differentiated by degrees of vulnerability, and presenting components of social phenomena such as inequality and heterogeneity in the labor market.

Key words: Social security, pensions, State of Mexico, aging, multiple correspondence analysis. 


\section{INTRODUCCIÓN}

$\mathbf{E}$

n las últimas décadas se han presentado cambios en la dinámica demográfica que impactan en múltiples aspectos de la población. Una de estas transformaciones ha sido el envejecimiento de la población que afecta a la sociedad a través de problemas de saturación en la atención a la salud y el financiamiento de las pensiones, entre otras.

En términos demográficos el siglo XXI se caracteriza por el constante y el sostenido crecimiento porcentual de la población de 60 años o más. Este aumento poblacional es también llamado envejecimiento demográfico, ${ }^{21}$ que es un proceso evidente en la mayoría de las sociedades latinoamericanas, y se debe fundamentalmente a la confluencia de dos factores: el descenso de la mortalidad y de la fecundidad.

En América Latina, el envejecimiento demográfico se ha vinculado con situaciones sociales complejas para este grupo poblacional. En particular en México, las personas envejecidas se han asociado con un deterioro de las condiciones de vida, debido a la falta de protección social. Según Vázquez (2013), una de las principales problemáticas asociadas al envejecimiento en México es la limitada cobertura de seguridad social en los adultos mayores. En particular, en el país para el año 2015, una de cada dos personas mayores de 60 años carecen de seguridad social (INEGI, 2015). En el caso del Estado de México, la situación de las personas adultas mayores es similar al nivel nacional, con 50 por ciento de los mexiquenses envejecidos, sin acceso a la seguridad social, lo que implica una vejez asociada a múltiples carencias. Ante este escenario, la presente investigación pretende analizar la situación de la seguridad social de los trabajadores asalariados mexiquenses de 50 a 59 años que se encuentra en Transición al Envejecimiento, con la finalidad de prever en qué condiciones de seguridad social se incorpora este grupo a la adultez mayor en el año 2025.

En particular, el objetivo de esta investigación es analizar la relación de la seguridad social con las características sociodemográficas y laborales de los trabajadores asalariados de 50 a 59 años, en el Estado de México.

En el Estado de México, la problemática del envejecimiento demográfico se ha abordado a través del diseño e implementación de políticas públicas vinculadas con apoyos monetarios, o en especie, para los adultos mayores (Gente Grande, Adultos en Grande, 60-69, 70 y más, entre otros).

${ }^{21}$ Para esta investigación el envejecimiento se denota como el aumento en términos absolutos y porcentuales de la población de 60 años y más respecto al total de la población. 
Sin embargo, estos apoyos gubernamentales son limitados debido a que no consideran del todo las condiciones de vida de los individuos en este grupo poblacional, como el deterioro funcional y el deterioro cognoscitivo de los mismos, así como condiciones estructurales relacionadas con el desempleo y la baja cobertura de seguridad social, elementos que inciden en la acumulación del déficit social y en los niveles de vida de este grupo de población.

Lo anterior se agudiza con el actual modelo económico neoliberal, que ha generado la erosión de los sistemas de protección social, trasladando los múltiples costos de la vejez directamente a los individuos (Castel, 2006), y sus familias. En particular las personas envejecidas se convierten en un grupo vulnerable, debido a que la baja cobertura de seguridad social incrementa sus riesgos de impactar negativamente en condiciones sociales y económicas de los adultos mayores.

En este contexto, el artículo contiene cinco apartados. En primer lugar, se presenta una revisión conceptual de Seguridad social. En segundo lugar, se muestra un breve contexto de la seguridad social en México, y el Estado de México. En tercer lugar, se expone la metodología, que consiste en realizar un Análisis de Correspondencia Múltiple. En cuarto lugar, se muestra la aplicación, y los resultados del análisis de correspondencia múltiple. Por último, se presentan las conclusiones.

\section{Revisión conceptual de Seguridad Social}

El concepto de Seguridad social tiene sus orígenes desde la época primitiva, cuando la población comenzó a agruparse en tribus o comunidades con la finalidad de enfrentar socialmente los múltiples riesgos naturales de aquellos tiempos. Para Nugent (1997) "el germen de la seguridad social lo encontramos entonces inscrito en la humanidad desde los tiempos más remotos. El hombre se enfrenta a un mundo que no entiende y que le agrede constantemente (...)" Nugent (1997: 603).

Después de estos primeros indicios de seguridad social, en Inglaterra en el siglo VII se implementó la ley de los pobres que se considera un primer antecedente institucional de la seguridad social. Esta ley consistía en que el Estado otorgaba protección social (limosna, asilo, trabajo, entre otras) a la personas necesitadas a través de las parroquias locales (Rodríguez, 2003).

Sin embargo fue hasta el año de 1889 , en Alemania, que por primera vez se implementa un modelo de seguridad social con el canciller Otto Von Bismarck. A este modelo se le denominó seguro social ${ }^{22}$ y consistía en otorgar un seguro que protegiera a los trabajadores en caso de enfermeda-

\footnotetext{
${ }^{22}$ Este seguro social fue financiado por los trabajadores y los empleadores.
} 
des, accidentes de trabajo o retiro. Es importante señalar que se concedió este beneficio, debido a las presiones de las organizaciones obreras que reclamaban un cambio de sistema político y económico (Santín y Alemán, 2009).

Con la implementación del seguro social en Alemania se expande la idea de otorgar este beneficio en diversos países, con el objetivo de que los trabajadores contaran con una protección social que permitiera mejorar su nivel de vida. En América Latina, los primeros países que implementaron este modelo de protección social fueron Brasil, Argentina y Chile. En el caso de México, la primera iniciativa de ley del seguro social se presentó en 1925 (Díaz, 2000).

Con base en lo anterior, el modelo de seguridad social se afianzaba, y en el año de 1942 se presentó el plan de seguridad social propuesto por Beveridge, que planteaba la protección de todos los ciudadanos a través de un proyecto completo de seguros (Sánchez, 2012).

A grandes rasgos, el seguro social propuesto por Bismarck, y el plan de seguridad social de Beveridge, fueron la base del modelo de seguridad social que rige hoy en día en casi todos los países. Estos modelos posicionaron el concepto de seguridad social a nivel internacional, de tal forma que desde el año de 1948 la seguridad social se considera un derecho humano. Cuando la Organización de las Naciones Unidas (ONU) plantea que "Toda persona, como miembro de la sociedad, tiene derecho a la seguridad social" (ONU, 1948: 43). Para la Organización Internacional del Trabajo (OIT, 2012), este derecho otorga seguridad económica, atención médica, prestaciones laborales, elementos que resultan esenciales para el bienestar de la población envejecida.

En términos conceptuales, la OIT (1984) define a la seguridad social como "la protección que la sociedad otorga contra las contingencias económicas y sociales derivadas de la pérdida de ingresos a consecuencia de enfermedades, maternidad, riesgos de trabajo, invalidez, vejez y muerte, incluyendo la asistencia médica" (OIT, 1984: 48).

Al respecto, Bertranou (2005), concibe a la Seguridad Social como

todo régimen o programa establecido por ley o por cualquier disposición obligatoria que garantiza una protección, sea a través de prestaciones en dinero o en especie, en caso de accidentes de trabajo, enfermedades profesionales, desempleo, maternidad, enfermedad común, invalidez, vejez, jubilación, sobrevivencia o muerte, e incluye, entre otros, prestaciones por hijos y por otros miembros de la familia, prestaciones de salud, prevención, rehabilitación y cuidados de larga duración. El término puede incluir al seguro social, a la asis- 
tencia social, a los regímenes de prestaciones mutuales, a los fondos de previsión y a los otros regímenes especiales (Bertranou, 2005:36).

Para este autor además la seguridad social incluye aquellas formas en las que instituciones privadas otorgan sistemas de ahorro o de seguros médicos y prestaciones como guarderías, escuelas, etcétera.

En contraste con las posturas anteriores, la Asociación Internacional de la Seguridad Social (AISS) plantea que la seguridad social debe contar, como elemento central de protección, con la seguridad económica de los individuos, con la finalidad de cubrir eventualidades como el retiro, las enfermedades, los accidentes, entre otros.

Como se expone anteriormente, el debate del concepto de seguridad social se centra en los beneficios que debe otorgar este modelo de protección social. Según Ham (1996), la seguridad social no debe reducirse sólo a la seguridad económica, y debe considerarse que la intención primordial de cualquier concepción de seguridad social debe buscar la estabilidad de la población en los diferentes aspectos de la vida cotidiana, y es en relación con ello que además de las pensiones resulta necesario incluir y analizar los rubros de la derechohabiencia y la salud.

En este contexto, la seguridad social se plantea como eje analítico de este artículo, debido a que interesa analizar la situación de la protección social de los trabajadores asalariados de 50 a 59 años, en la entidad mexiquense, ante un proceso de envejecimiento acelerado.

En las líneas anteriores se presentaron algunos planteamientos sobre el concepto de seguridad social, destacando la falta de consenso pleno en la definición del mismo, así como en sus elementos conceptuales. Sin embargo, con la revisión de la bibliografía muestra suficiente evidencia para señalar algunos factores relevantes de la seguridad social, como la seguridad económica y la atención médica. Estos elementos se vinculan con el acceso a las pensiones y la derechohabiencia. Por lo anterior, para esta investigación la seguridad social consta de dos aspectos trascendentales: las pensiones y la derechohabiencia. La elección de estos elementos se asocia con aspectos económicos y de salud que resultan vitales para mejorar las condiciones materiales y el nivel de vida de esta población.

Las pensiones se asocian directamente con la seguridad económica de los adultos mayores, debido a que otorgan elementos a esta población para lograr condiciones de vida adecuadas (CEPAL, 2008). Los recursos financieros en la vida de las personas adultas mayores se vinculan con la satisfacción de necesidades, la toma de decisiones, y la participación en la vida cotidiana. 
En lo que respecta a la derechohabiencia, de acuerdo con Barié (2003), la población derechohabiente es aquella que se encuentra afiliada a alguna institución de seguridad social ${ }^{23}$, con ello tiene garantizado el acceso a un conjunto de prestaciones, entre las que se encuentran los servicios médicos. Uno de los beneficios de la derechohabiencia consiste en cubrir los gastos de la atención médica del trabajador, y de sus familiares, en la institución pública o privada donde el trabajador se encuentra afiliado.

Acorde con lo anterior, en esta investigación se eligieron como variables empíricas el acceso a las pensiones y la derechohabiencia, debido a que permiten estimar la seguridad social, y conocer su relación con las características sociodemográficas y laborales de los trabajadores asalariados de 50 a 59 años mexiquenses.

\section{Seguridad social en MéXico y el Estado de MéXico}

$\mathrm{Al}$ concluir la primera guerra mundial diversos países retomaron los sistemas de seguridad social alemanes ${ }^{24} q u e$ promovían la protección y el bienestar de los trabajadores con la finalidad de mitigar la indefensión que aquejaban a dicha población (Díaz, 2000). Esta política social influyó en varios países como México, que presentaba en las primeras décadas del siglo XX preocupaciones asociadas con la implementación de un sistema de seguridad social.

En 1925, en México, se presentó una iniciativa de Ley sobre accidentes de trabajo y enfermedades profesionales, que cubría únicamente al sector patronal. Para 1929 se crea la Ley del Seguro Social que contenía diversos seguros asociados con la invalidez, la cesación involuntaria del trabajo, las enfermedades, los accidentes, entre otros. Sin embargo, hasta el año de 1942 se implementó dicha Ley, motivada por las circunstancias de la segunda guerra mundial, y las desigualdades económicas y sociales que se incrementaban en el país (Díaz, 2000). Finalmente, en 1943 se publicó en el Diario Oficial de la Federación la Ley del Seguro Social, que ha beneficiado a millones de trabajadores y a sus familias.

A pesar de que en la actualidad la seguridad social otorga beneficios relevantes a los trabajadores mexicanos, uno de las problemáticas que enfrenta la seguridad social es el financiamiento de este sistema. Lo anterior

\footnotetext{
${ }^{23}$ En el caso de México las instituciones que otorgan seguridad social son: el Instituto Mexicano del Seguro Social (IMSS), el Instituto de Seguridad Servicios Sociales de los Trabajadores del Estado (ISSSTE), el ISSSTE a nivel estatal, el Instituto de Seguridad Social para las Fuerzas Armadas Mexicanas (ISSFAM), entre otros.

${ }^{24}$ Los sistemas de seguridad social alemanes fueron diseñados por Otto Von Bismarck y consistían en un programa de indemnización y un seguro de enfermedad para los trabajadores alemanes de esa época (OIT, 2009).
} 
se debe a múltiples cambios económicos, sociales y demográficos que han afectado la dinámica y la cobertura de la seguridad social en México.

\section{El contexto del envejecimiento demográfico en el Estado de México}

A mediados de la segunda década del siglo XXI, el Estado de México se encuentra en la fase final de su transición demográfica, que se prevé termine en corto plazo. Como resultado de este proceso, la entidad mexiquense tendrá, en tan sólo un par de décadas, porcentajes significativos de población envejecida, lo que hace evidente la necesidad de analizar la seguridad social con la finalidad de conocer en qué situación se encuentran las personas de 50 a 59 años, que son quienes se incorporarán al grupo de adultos mayores mexiquenses en el corto plazo.

En el año 2015 los adultos mayores ${ }^{25}$ en el Estado de México eran aproximadamente 1.5 millones de personas, que representaban casi 10 por ciento de la población total. Es decir, uno de cada diez mexiquenses eran personas envejecidas. Este porcentaje se prevé que aumente en las próximas décadas. Según el Consejo Nacional de Población (CONAPO), la población envejecida en el Estado de México en el año 2030 será de casi tres millones de personas, es decir, 14 por ciento de la población mexiquense estará en edades avanzadas.

Ante esta situación demográfica resulta interesante conocer a la población en Transición hacia el Envejecimiento (TE), debido a que este grupo de personas engrosarán las filas de los adultos mayores en los años venideros. Para esta investigación, las personas en TE se definen como aquellas que en el año 2015 tenían entre 50 y 59 años. Una de las razones de estudiar este grupo poblacional radica en conocer la situación de la seguridad social, a través de características sociodemográficas y laborales de esta población, que en años futuros se incorporará al grupo poblacional de los adultos mayores. Lo anterior permite estudiar los aspectos sociales y económicos que impactan en la vulnerabilidad social de este grupo, e impiden garantizar una vejez digna a los mexiquenses que se encuentran en la antesala del envejecimiento (INEGI, 2015). Es importante destacar, que en los próximos años, el envejecimiento de la población mexiquense representará un fenómeno social de relevancia por las complejidades sociales que conllevan, por lo cual sería indispensable contar con un sistema de protección social que permita desarrollar en la vejez un nivel de vida digno para esta población.

\footnotetext{
${ }^{25}$ Para esta investigación los adultos mayores son las personas que tienen 60 años o más.
} 
En este contexto, en la Tabla 1 se expone el crecimiento constante del número de personas mayores de 60 años a nivel nacional y en el Estado de México. Por ejemplo, en el año 2000 a nivel nacional la población adulta mayor era de casi siete millones de personas, para 2010 este grupo poblacional ya contaba con diez millones, y se espera que en el año 2030 alcance la cifra de 20 millones de personas adultas mayores en México. En lo que respecta al Estado de México el incremento de personas envejecidas es relevante, debido a que entre 1990 y 2030 la población adulta mayor se incrementará anualmente en 4.8 por ciento; lo que expone una situación compleja para los actores sociales mexiquenses.

Tabla 1: Población de adultos mayores en México y en el Estado de México, 2000 al 2030

\begin{tabular}{lrrrrrrr}
\hline \multirow{2}{*}{ Entidad } & 1990 & 2000 & 2010 & 2015 & 2020 & 2030 \\
\cline { 2 - 7 } & 4988158 & 6948457 & 10055379 & 12436321 & 14425879 & 20365839 \\
\hline México & 450921 & 713704 & 1137647 & 1517425 & 1917767 & 2972420 \\
Estado de México & $\begin{array}{l}\text { Nota: n/d = No disponible. } \\
\text { Fuente: Censos de Población y Vivienda de México 1990, 2000 y 2010, Encuesta Intercensal 2015, y Proyecciones de población } \\
\text { del INEGI. }\end{array}$
\end{tabular}

Ante este escenario de un envejecimiento acelerado, en las siguientes líneas se presentan las características sociodemográficas y laborales de la población mexiquense de 50 a 59 años, con la finalidad de contextualizar a las personas en transición a la vejez mexiquense. Lo anterior se presenta como preámbulo al análisis de la situación de la seguridad social de este grupo poblacional.

En el año 2015, el volumen de la población mexiquense de 50 a 59 años era de aproximadamente 1.5 millones, de los cuales 52 por ciento eran mujeres, y 48 por ciento hombres (Tabla 2). De este grupo de población, casi 56 por ciento se encuentra entre los 50 y 54 años de edad, y 44 por ciento entre los 55 y 59 años, para esta variable las diferencias por sexo son mínimas. Lo anterior expone que la población en transición a la adultez mayor representa un número importante de personas, que podrían sobre saturar los sistemas de seguridad social en el Estado de México al llegar a formar parte de los adultos mayores.

En relación al nivel educativo, en el año 2015 más de 60 por ciento de esta población sólo tiene educación básica. En el nivel medio superior (preparatoria y bachillerato) alcanzaban más de 12 por ciento. Para el nivel profesional y de posgrado la población de 50 a 59 años mexiquenses era de 13 por ciento (Tabla 2 ). 
Tabla 2: Distribución porcentual de indicadores sociodemográficos de personas de 50 a 59 años según sexo, Estado de México, 2015

\begin{tabular}{lrrr}
\hline $\begin{array}{l}\text { Indicadores sociodemográficos } \\
\text { seleccionados }\end{array}$ & Hombres & Mujeres & Total \\
\hline Grupos de edad & & & \\
50 a 54 & 57.1 & 56.7 & 56.9 \\
55 a 59 & 42.9 & 43.3 & 43.1 \\
Nivel educativo & & & \\
$\quad$ Ninguno & 3.7 & 7.3 & 5.6 \\
Básico & 60.1 & 63.9 & 62.1 \\
Preparatoria y bachillerato & 15.6 & 9.2 & 12.2 \\
Normal y carrera técnica & 4.2 & 9.6 & 7.1 \\
Profesional y Posgrado & 16.2 & 9.8 & 12.8 \\
No sabe & 0.1 & 0.1 & 0.1 \\
Estado conyugal & & & \\
Unión libre & 14.3 & 9.2 & 11.6 \\
Separado & 4.5 & 9.4 & 7.1 \\
Divorciado & 2.0 & 3.6 & 2.9 \\
Viudo & 2.5 & 9.8 & 6.4 \\
Casado & 71.1 & 59.4 & 64.9 \\
Soltero & 5.5 & 8.2 & 6.9 \\
No especificado & 0.2 & 0.2 & 0.2 \\
Total & 727007 & 817439 & 1544446 \\
\hline Funte elborán propia & &
\end{tabular}

Fuente: elaboración propia con base en la Encuesta Intercensal 2015, INEGI.

Un elemento que sobresale en la variable de nivel educativo son las diferencias por sexo. En la Tabla 2 se expone que en el año 2015 las mujeres tenían desigualdades educativas relevantes, debido a que tenían una mayor concentración en ningún nivel educativo, y en educación básica. En contraste, en los niveles educativos altos se concentraban mayoritariamente los hombres, lo que confirma las desigualdades históricas que enfrentan las mujeres en los aspectos educativos (Ariza y De Oliveira, 2000).

Es importante señalar que diversos autores (Mora, 2012; Guadarrama, 2012) muestran que existe una fuerte relación entre el bajo nivel educativo, y las adversas condiciones laborales. Estos vínculos se acentúan en un mercado laboral como el mexiquense, en el que según Villaseñor (2016) más de 60 por ciento de los trabajadores asalariados cuentan con trabajos 
precarios. Es decir, en el Estado de México el acceso a la seguridad social está fuertemente limitado por las desfavorables condiciones que enfrentan los trabajadores.

En la última parte de la Tabla 2 se presenta la variable de situación conyugal, en el año 2015, en donde un poco más de 75 por ciento de los mexiquenses de 50 a 59 años se concentraban en la categorías de casados o unidos. Es decir, tres de cada cuatro mexiquenses en transición hacia el envejecimiento tenían una relación de pareja. En contraste, 25 por ciento de este grupo poblacional son separados, viudos o solteros.

Lo anterior expone que en el año 2015, la población mexiquense de 50 a 59 años concentra un mayor número de mujeres, más de la mitad sólo tenía baja educación, y casi 75 por ciento están casados o unidos.

Una vez que se exponen las características sociodemográficas para conocer con mayor detalle a la población mexiquense de 50 a 59 años, en la Tabla 3 se presenta la condición de actividad de este grupo poblacional para saber qué tipo de labor realizan estas personas antes de incorporarse al envejecimiento.

Tabla 3: Distribución porcentual de la condición de actividad de las personas de 50 a 59 años del Estado de México, 2015

\begin{tabular}{lrrr}
\hline Condición de actividad & Hombres & Mujeres & Total \\
\hline Trabajo & 82.4 & 36.8 & 58.3 \\
Busca trabajo & 3.4 & 0.3 & 1.8 \\
Estudiante & 0.0 & 0.1 & 0.1 \\
Jubilado o pensionado & 4.4 & 3.4 & 3.9 \\
Quehaceres del hogar & 0.9 & 55.0 & 29.5 \\
Limitación física o mental & 1.9 & 1.0 & 1.4 \\
No trabajo & 6.7 & 2.9 & 4.7 \\
No especificado & 0.2 & 0.4 & 0.3 \\
Total & 727007 & 817439 & 1544446 \\
\hline
\end{tabular}

Fuente: elaboración propia con base en la Encuesta Intercensal 2015, INEGI.

En la Tabla 3 se expone que en el año 2015, casi 60 por ciento de la población mexiquense de 50 a 59 años se encontraba inserta en el mercado laboral, seguido de quienes se dedican a los quehaceres del hogar, con aproximadamente 30 por ciento, el otro 10 por ciento se distribuye entre las otras categorías. Las diferencias por sexo en la condición de actividad son relevantes, debido a que en este grupo de edad los hombres predomi- 
nan en el mercado laboral, con una participación de 80 por ciento. En contraste, sólo 36 por ciento de las mujeres trabajan. En lo que respecta a los quehaceres del hogar, 55 por ciento de las mujeres participan en esta actividad, contra sólo uno por ciento de los hombres. Lo anterior expone que las diferencias por sexo en este grupo de edad se agudizan, y reproducen las desigualdades históricas en los roles sociales de los hombres y las mujeres.

Una vez que se expone la condición de actividad de los mexiquenses de 50 a 59 años, en las siguientes líneas se analiza la población objetivo de esta investigación, que son los trabajadores asalariados de 50 a 59 años del Estado de México. Es importante señalar que en México la seguridad social es un beneficio que históricamente ha estado asociado a los trabajadores asalariados.

En este sentido, según la Encuesta Intercensal, en el año 2015 había casi 800 mil trabajadores asalariados, de los cuales aproximadamente 66 por ciento están afiliados a alguna institución de salud. Esto plantea que la cobertura de la derechohabiencia en el Estado de México tiene serias deficiencias, debido a que cuatro de cada diez trabajadores asalariados mexiquenses en TE no cuentan con los servicios que otorga esta afiliación, y en los próximos años el escenario se agudiza porque esta población se incorporará a la adultez mayor, que puede requerir de mayores servicios de salud. Una vez que se expone a grandes rasgos la problemática de la derechohabiencia en el Estado de México, con la población de 50 a 59 años, en las siguientes líneas se plantean los aspectos relacionados con el acceso a las pensiones.

Las pensiones se definen como un ingreso económico que permite a las personas mantener un nivel de consumo, al término o a la interrupción de la vida laboral (López, 1987). Sin embargo, en México y el Estado de México el sistema previsional tiene múltiples deficiencias, como la escasa cobertura y los bajos ingresos por pensiones, que al final impactan en el nivel de vida de las personas que se retiran del trabajo (Pedrero, 1999; Salas, 1999). En 2015, de acuerdo con datos de la Encuesta Intercensal, la población en transición al envejecimiento que contaba, por su trabajo, con ahorro para el retiro era de 50 por ciento (INEGI, 2015).

Lo anterior plantea que en el mejor de los casos, en el año 2025, sólo la mitad de la población mexiquense de 50 a 59 años recibirá pensión. Es importante señalar que el sistema de pensiones que prevalecerá en ese año será el de cuentas individuales, que tiene entre sus principales proble- 
máticas una baja tasa de reemplazo, ${ }^{26}$ por lo que en la próxima década la situación se complejiza para los adultos mayores, debido a que los ingresos por pensiones serán precarios, y eso sólo para aquellos que sí estén pensionados.

Adicionalmente a las situaciones descritas, relacionadas con las pensiones y la derechohabiencia, los adultos en TE se enfrentan a retos importantes relacionados con la salud. Esto cobra importancia por dos motivos: por un lado, la derechohabiencia en la población mencionada se encuentra segmentada, mientras que por otro lado, una salud comprometida incrementa los gastos en salud, impactando la vulnerabilidad de los individuos y las familias (OMS, 2011).

La población mexicana de 50 a 59 años posee un perfil de salud caracterizado por la coexistencia de padecimientos crónicos y transmisibles, con malnutrición (Shamah-Levy et al., 2008). Particularmente, padecimientos como la diabetes mellitus o la hipertensión arterial se han incrementado en menores de 60 años (Instituto Nacional de Salud Pública - INSP, 2016). Entre las consecuencias del incremento de la diabetes mellitus o la hipertensión arterial se encuentran: el aumento de los riesgos de mortalidad, una mayor dependencia funcional, o el incremento de los gastos relacionados con la salud (Manrique-Espinoza et al., 2013).

Como se comenta en líneas anteriores, la población mexicana de 50 a 59 años se enfrenta a importantes problemas de salud. En el caso de la población mexiquense, resultados de la Encuesta Nacional de Salud y Nutrición (ENSANUT) 2012, indican que la prevalencia de hipertensión arterial en mujeres de 60 y más años es 40.5 por ciento y 37.7 por ciento en hombres, lo que es 9.9 veces la prevalencia de las personas de 20-30 años. De manera similar, la prevalencia de diabetes mellitus en mujeres de 40 a 59 años es de 12.2 por ciento y de 19.3 por ciento en hombres, aumentando a 30.3 por ciento en mujeres mayores de 60 años y a 25.5 por ciento en hombres. En este sentido, la prevalencia de diabetes mellitus en la población mexiquense se encuentra por encima de la media nacional (10.6 por ciento para el Estado de México, y 9.2 por ciento para el país).

Ante esta situación, el gobierno, la sociedad, y las personas que se van a integrar a los adultos mayores mexiquenses en la próxima década enfrentan un reto importante en materia de salud, que podría mitigarse a través del diseño y la implementación de políticas sociales que garanticen el acceso a la seguridad social de esta población. Para contribuir en lo anterior,

${ }^{26}$ La tasa de reemplazo es el porcentaje de la pensión con respecto al salario que recibe un trabajador jubilado. 
esta investigación utiliza el Análisis de Correspondencia Múltiple, el cual permite identificar grupos poblacionales que presenten vulnerabilidad, limitantes o falta de acceso a la seguridad social.

\section{CONSIDERACIONES METOdológICAS}

En este apartado se abordan los aspectos metodológicos que se utilizan para analizar, en el año 2015, la situación de la seguridad social de los trabajadores asalariados mexiquenses de 50 a 59 años, a través de sus características sociodemográficas y laborales. En particular se utilizan dos elementos de la seguridad social (acceso a las pensiones y la derechohabiencia).

\section{Metodología}

La metodología que se utiliza en esta investigación consiste en la aplicación del Análisis de Correspondencia Múltiple (ACM). Esta técnica estadística permite analizar la relación entre diversas variables categóricas, de tal forma que se puedan conformar grupos dependiendo de la cercanía / lejanía de las categorías que se observen en la gráfica del ACM (Greenacre, 1993). Este método ayuda a explorar los datos y permite conocer los principales vínculos que se generen a partir de la tabla. La elección del ACM se debe al objetivo de este artículo, que plantea analizar la relación entre la seguridad social y las características sociodemográficas y laborales de los trabajadores asalariados mexiquenses de 50 a 59 años. Para este análisis empírico las variables que se asocian con la seguridad social son el acceso a las pensiones y la condición de derechohabiencia. Con respecto a las características sociodemográficas, las variables que se utilizan son: sexo, grupos de edad, nivel educativo, condición migratoria y situación conyugal. Para las características laborales se utilizan los grupos ocupacionales y el ingreso por trabajo.

\section{Análisis de Correspondencia Múltiple}

El ACM es una técnica estadística que se utiliza para estudiar la relación entre variables categóricas. Según Greenacre (1993) "la principal meta del análisis de correspondencia es transformar una tabla de información numérica en una visualización gráfica" (Greenacre, 1993: 58).

La población objetivo de esta investigación son los trabajadores asalariados de 50 a 59 años en el Estado de México. La elección de este grupo poblacional se debe a tres aspectos. En primer lugar, la acotación del gru- 
po de edad se debe a que esta población en el año 2025 será parte de los adultos mayores, por lo que permite prever a grandes rasgos la situación de seguridad social, y delimitar algunas problemáticas de protección social que se puedan presentar en este conjunto de personas. En segundo lugar, la elección de los trabajadores asalariados se debe a que esta población son los que tienen acceso a la derechohabiencia y a las pensiones. Por último, el Estado de México se seleccionó debido a que en la actualidad esta entidad tiene un proceso de envejecimiento acelerado, el cual en la próxima década se acentuará con la incorporación de aproximadamente 1.5 millones de personas a la adultez mayor.

\section{Fuente de información}

La fuente de información que se utiliza en esta investigación es la Encuesta Intercensal (EI) ${ }^{27} 2015$, que tiene como objetivo generar información estadística actualizada, que proporcione estimaciones con calidad sobre el volumen, la composición, y la distribución de la población y de las viviendas del territorio nacional, que mantenga la comparabilidad histórica con los censos y encuestas nacionales, así como con indicadores de otros países (Instituto Nacional de Estadística y Geografía - INEGI, 2015). Esta encuesta contiene información asociada a la seguridad social de la población, en particular cuenta con preguntas sobre el acceso a pensiones y la cobertura de la derechohabiencia. La representatividad de esta fuente de datos es a nivel nacional, estatal, y municipal.

Según el documento metodológico de la Encuesta lntercensal 2015 (INEGI, 2015), el tamaño de muestra que se utilizó en este levantamiento fue de 5.9 millones de viviendas habitadas, y "el esquema de muestreo fue estratificado por conglomerados y en una sola etapa, es decir, para formar los estratos se clasificaron las localidades según tamaño y nivel socioeconómico, se seleccionaron áreas geográficas completas utilizando muestreo aleatorio simple, y en su interior, se visitaron todas las viviendas para captar sus características y las de sus residentes" (INEGI, 2015: 6).

La elección de esta fuente de información se debe a que la EI cuenta con información sobre los elementos conceptuales de la seguridad social, que se plantean analizar en esta investigación: acceso a pensiones y condición de derechohabiencia. Además, el año de levantamiento de esta encuesta permite analizar a la población en estudio, y estimar la situación de la seguridad social de este grupo poblacional en el año 2025, cuando éstos sean adultos mayores, es decir, diez años después. Es importante señalar que la

\footnotetext{
${ }^{27}$ Esta encuesta fue hecha por el Instituto Nacional de Estadística y Geografía (INEGI).
} 
EI sustituyó a los conteos de población que se realizaban cada cinco años en México.

En la Tabla 4, se exponen las preguntas de la EI que logran captar las dos variables de seguridad social. En lo que respecta al acceso a las pensiones la pregunta hace referencia al ahorro para el retiro a través del $\mathrm{SAR}^{28} \mathrm{o}$ la AFORE ${ }^{29}$ que son los mecanismos que administran las pensiones de los trabajadores. Para la pregunta sobre derechohabiencia, en las respuestas se incluye el Seguro Popular ${ }^{30}$ (SP), sin embargo, para esta investigación este seguro no se considera como un elemento inherente a la derechohabiencia, ya que se vincula con aspectos de asistencia social, y la derechohabiencia es un derecho laboral.

Tabla 4: Preguntas asociadas a las variables de acceso a las pensiones y de derechohabiencia de la Encuesta Intercensal 2015

¿(NOMBRE) tiene por su trabajo: SAR o AFORE (ahorro para el retiro)?
¿(NOMBRE) está afiliada(o) o tiene derecho a los servicios médicos en:

Seguro Popular o para una Nueva Generación (Siglo XXI)?

IMSS (Seguro Social)? ISSSTE?

ISSEMYM?

Pemex, Defensa o Marina?

Seguro privado?

Otra institución?

Entonces, ¿no está afiliada(o)a servicios médicos?

Fuente: cuestionario de la Encuesta Intercensal, 2015.

Aunado a lo anterior, la cobertura del SP tiene dos principales diferencias con la atención médica que se otorga por afiliación a una institución de salud específica. Por un lado, el tipo de atención médica en la derechohabiencia permite atender enfermedades especializadas, que frecuentemente se presentan en la población envejecida, situación que no sucede en el SP. Por el otro, el servicio médico que otorga la seguridad social cubre desde el primer nivel de atención, mientras que el SP principalmente se enfoca la atención de primer nivel en centros de salud.

\footnotetext{
${ }^{28}$ Sistema de Ahorro para el Retiro.

${ }^{29}$ Administradora de Fondos para el Retiro.

${ }^{30} \mathrm{http}$ ://www.seguropopular.org/
} 
Es importante señalar que en México existen pocas fuentes de información especializadas en la seguridad social. La principal encuesta asociada a este fenómeno es la Encuesta Nacional de Empleo y Seguridad Social (ENESS). Sin embargo esta encuesta tiene la limitante de que la última disponible se realizó en el año 2013.

\section{Aplicación de Análisis de Correspondencia Múltiple}

Una vez esbozado el contexto general de la población del Estado de México, así como la situación de la seguridad social de la población objetivo de esta investigación, en las siguientes líneas se expone la aplicación del $\mathrm{ACM}$, que permite formar grupos de población, con base en la relación de la seguridad social (derechohabiencia y pensiones), y las características sociodemográficas y laborales. En este sentido, según Bordieu (2009), el $\mathrm{ACM}$ es una herramienta que expone gráficamente un mapa social que es representado por las categorías de diferentes variables, y entre más cercanas estén las categorías, mayor relación existe entre las variables. Antes de iniciar el análisis de la seguridad social se presentan las variables que se utilizaron en el ACM, con la descripción de cada una de las categorías, con la finalidad de que, al observar la Figura 1, se comprenda con mayor claridad las relaciones estudiadas en esta investigación.

La Figura 1 muestra el ACM en el año 2015, aplicado a los trabajadores asalariados de 50 a 59 años del Estado de México. Este ACM permite distinguir tres conjunto de trabajadores que presentan diferencias por niveles educativos, ingresos por trabajo, ocupaciones, condición de derechohabiencia, y acceso a las pensiones. Entre estos conjuntos de población sólo dos grupos presentan diferencias marcadas por la seguridad social. Es importante señalar que estos tres grupos que se identificaron en el ACM se analizaron a través de grados de vulnerabilidad ${ }^{31}$ hacia la condición de seguridad social, debido a que la vulnerabilidad se asocia con el riesgo que enfrentan los trabajadores asalariados en TE al incorporarse a la población envejecida.

Al interior de la Figura 1, el grupo 1 está conformado por mujeres, viudas o solteras sin nivel educativo, bajos salarios, y trabajos sin calificación. Este grupo presenta características sociodemográficas asociadas con rezagos sociales y características laborales altamente precarias.

\footnotetext{
${ }^{31}$ En esta investigación se reconoce que el concepto de vulnerabilidad es amplio y complejo, sin embargo, para esta investigación sólo se utiliza de manera indicativa, debido a que lo que interesa analizar es la seguridad social.
} 
Tabla 5: Descriptor de variables del Análisis de Correspondencia Múltiple

\begin{tabular}{|c|c|}
\hline Variable & Descripción de la variable \\
\hline \multicolumn{2}{|l|}{ Sexo } \\
\hline $\mathrm{H}$ & Hombre \\
\hline M & Mujer \\
\hline \multicolumn{2}{|l|}{ Salario } \\
\hline $\begin{array}{l}>5 \mathrm{sm} \\
3 \text { a } 5 \mathrm{sm}\end{array}$ & $\begin{array}{l}\text { Mas de } 5 \text { salarios mínimos } \\
\text { de } 3 \text { a } 5 \text { salarios mínimos }\end{array}$ \\
\hline $1 \mathrm{a}<3 \mathrm{sm}$ & de uno a menos de 3 salarios mínimos \\
\hline$<1 \mathrm{sm}$ & Menos de un salarios mínimos \\
\hline \multicolumn{2}{|l|}{ Ocupación } \\
\hline nmancalif & No manual calificado \\
\hline nmanncalif & No manual no calificado \\
\hline mancalif & Manual calificado \\
\hline manncalif & Manual no calificado \\
\hline \multicolumn{2}{|l|}{ Afore } \\
\hline AF_S & Si tiene AFORE \\
\hline AF_N & No tiene AFORE \\
\hline \multicolumn{2}{|l|}{ Derechohabiencia } \\
\hline PEMEX & Pemex, Defensa o Marina \\
\hline SEG_PRIVADO & Seguro privado \\
\hline ISSSTE & ISSSTE O ISSEMYM \\
\hline IMSS & IMSS \\
\hline OTRO_SEG & Otra institución de derechohabiencia \\
\hline NO_AFIL_SALUD & No esta afiliado \\
\hline \multicolumn{2}{|l|}{ Edad } \\
\hline $50-54$ & De 50 a 54 años \\
\hline $55-59$ & De 55 a 59 años \\
\hline \multicolumn{2}{|l|}{ Nivel educativo } \\
\hline PROF & Profesional \\
\hline TEC_NORMAL & Normal y carrera técnica \\
\hline PREPA & Preparatoria y bachillerato \\
\hline BASICA & Educación básica \\
\hline NIN_EDU & Sin educación \\
\hline \multicolumn{2}{|l|}{ Residencia } \\
\hline MISMO_MUN & Reside en el mismo municipio \\
\hline OTRO_MUN & Reside en otro municipio \\
\hline \multicolumn{2}{|l|}{ Situación conyugal } \\
\hline CASADO & Casado o unión libre \\
\hline DIVOR_VIU & Divorciado, separado o viudo \\
\hline SOLTERO & Soltero \\
\hline
\end{tabular}




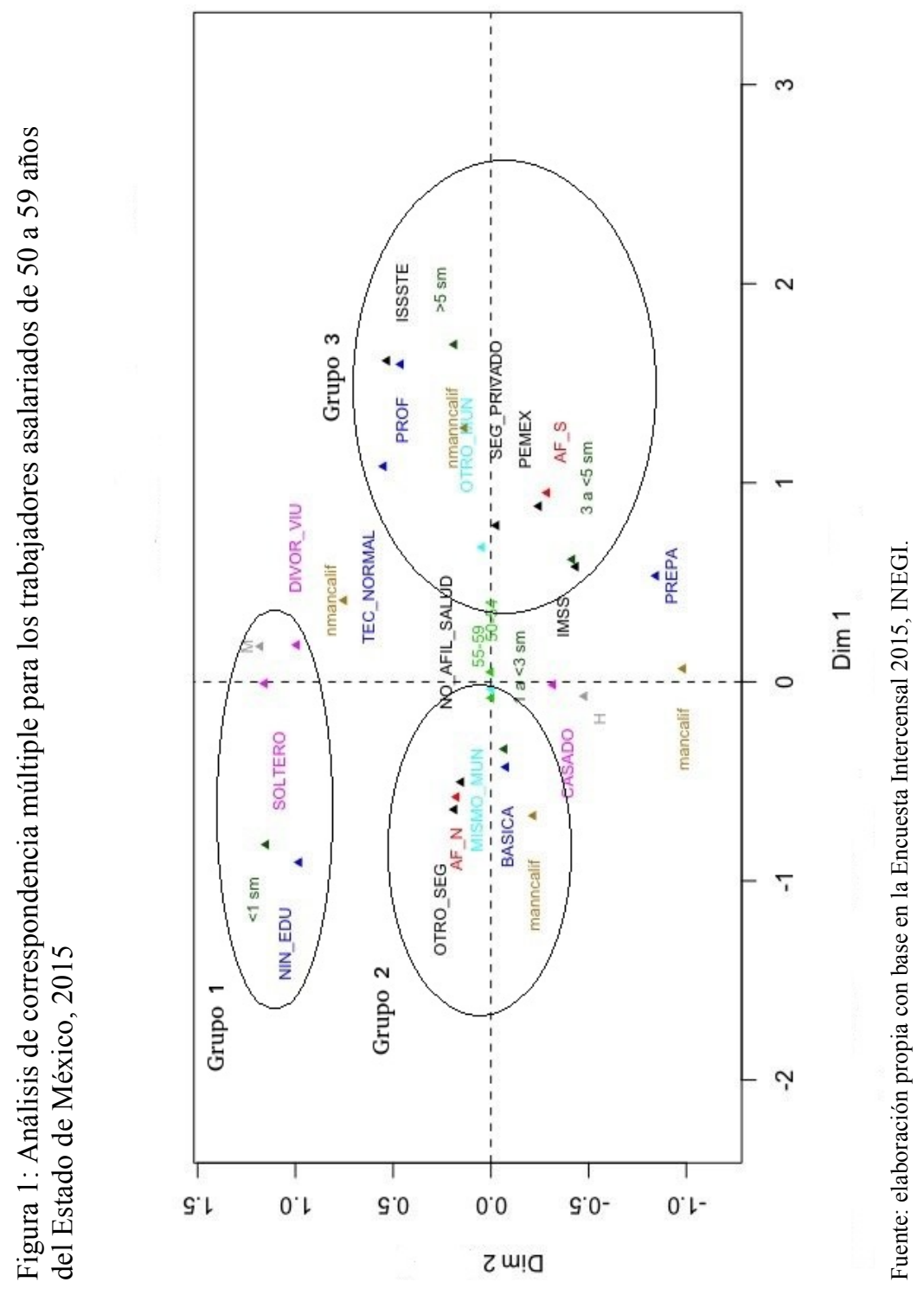


Con base en lo anterior, los trabajadores asalariados del grupo 1 son los que se identifican como altamente vulnerables, debido a que no tienen vínculos con la seguridad social, su capacidad de ahorro es casi nula, y las redes familiares son débiles. Esto conlleva a que en el año 2025, al incorporarse al envejecimiento, este conjunto de personas enfrentará el mayor riesgo de acceso a la seguridad social, ya que las relaciones de derechohabiencia y pensiones son nulas.

En lo que respecta al grupo 2, las características que predominan son educación básica, edades entre 55 a 59, no son migrantes, ingresos menores a tres salarios mínimos, laboran en comercio y servicios, restringida derechohabiencia, y sin acceso a pensiones (véase Figura 1). En este conjunto de trabajadores se identifica una clara relación entre los bajos niveles educativos, ingresos precarios, trabajos inseguros e inestables, con un limitado o nulo acceso a la derechohabiencia y a las pensiones, por lo que este grupo presenta condiciones de vulnerabilidad media hacia la seguridad social, lo que implica que al incorporarse al envejecimiento tendrán diversas restricciones en sus condiciones materiales y nivel de vida.

Para este grupo 2, una parte de estas problemáticas puede que sean absorbidas por las redes familiares, que en muchos casos son los que terminan como responsables de mantener o mejorar el nivel de vida de esta población. Es importante señalar que los adultos en TE son personas que trabajaron durante más de 30 años, por lo que es indispensable retribuirles los esfuerzos que realizaron durante su vida laboral a través de la garantía de seguridad social, que les permita mejorar o mantener su nivel de vida.

Por último, en la Figura 1 se presenta el grupo 3, que está conformado por los trabajadores que están mejor posicionados, en cuanto a los aspectos sociodemográficos, laborales, condición de derechohabiencia, y acceso a pensiones. El perfil de este grupo tiene las siguientes características: altos niveles educativos e ingresos, son migrantes, ${ }^{32}$ directivos o profesionistas, cuentan con derechohabiencia y pensiones.

En contraste con los grupos anteriores, el grupo 3 se considera como no vulnerable, debido a que cuenta con las mejores condiciones y relaciones (sociodemográficas, laborales, y de seguridad social) que les permita enfrentar, en el año 2025, su incorporación al envejecimiento demográfico.

En términos generales, la Figura 1 expone la importancia de la derechohabiencia y el acceso a las pensiones en la población objetivo, debido a que estas variables logran concentrar y dividen gran parte de las categorías

${ }^{32}$ La condición de migrantes calificados en el Estado de México se presenta por su colindancia con la ciudad de México donde existen grandes empresas que cuentan con trabajadores altamente calificados que residen la entidad mexiquense. 
analizadas en el ACM. Lo anterior muestra que en el año 2015, la seguridad social es un elemento diferenciador, con respecto a las características sociodemográficas y laborales, para los trabajadores asalariados de 50 a 59 años mexiquenses.

La presencia de tres grupos asociados con la vulnerabilidad de la población en estudio hacia la seguridad social permite plantear un esquema que muestra con mayor claridad el proceso que se llevó a cabo en esta investigación para analizar la situación de la seguridad social de los trabajadores asalariados de 50 a 59 años del Estado de México.

Para finalizar, la Figura 2 expone el eje analítico de esta investigación que corresponde a la seguridad social. En segundo plano se presentan los elementos empíricos que permitieron desarrollar la metodología del Análisis de Correspondencia Múltiple, y en los resultados se presentan los tres grupos que muestran gráficamente la relación entre la derechohabiencia y el acceso a pensiones, con las características sociodemográficas y laborales. En estos grupos se observan los riesgos que van a enfrentar las personas del Estado de México, que se incorporaran al envejecimiento en la próxima década.

\section{Conclusiones}

Las conclusiones de este artículo plantean que los trabajadores asalariados de 50 a 59 años del Estado de México presentan diversas problemáticas, que se agudizarán en el año 2025, cuando este conjunto de población se incorpore a los adultos mayores. En particular, los resultados de esta investigación exponen fragmentadas relaciones de la seguridad social con las características sociodemográficas y laborales. Lo que conlleva que una parte de la población en transición al envejecimiento del Estado de México enfrentará en la próxima década una vejez: sin seguridad social, redes familiares y sociales débiles, sin seguridad económica, entre otros.

Además, en el Análisis de Correspondencia Múltiple se observan diversos fenómenos sociales que complejizan el escenario a la población en transición al envejecimiento, debido a que la seguridad social funge como un factor que impacta en la desigualdad, al sólo ser beneficiados los trabajadores con mayores privilegios. En este sentido, para esta investigación, a partir de los resultados obtenidos se plantean cuatro conclusiones:

1. La relación entre la seguridad social, y las características sociodemográficas y laborales, expone una división entre aquellos que cuentan o no con esta primera. Esto plantea una fragmentación de la población en 
Situación de la seguridad social de los trabajadores asalariados de 50 a 59 años ... /S.C. GAXIOLA et al.

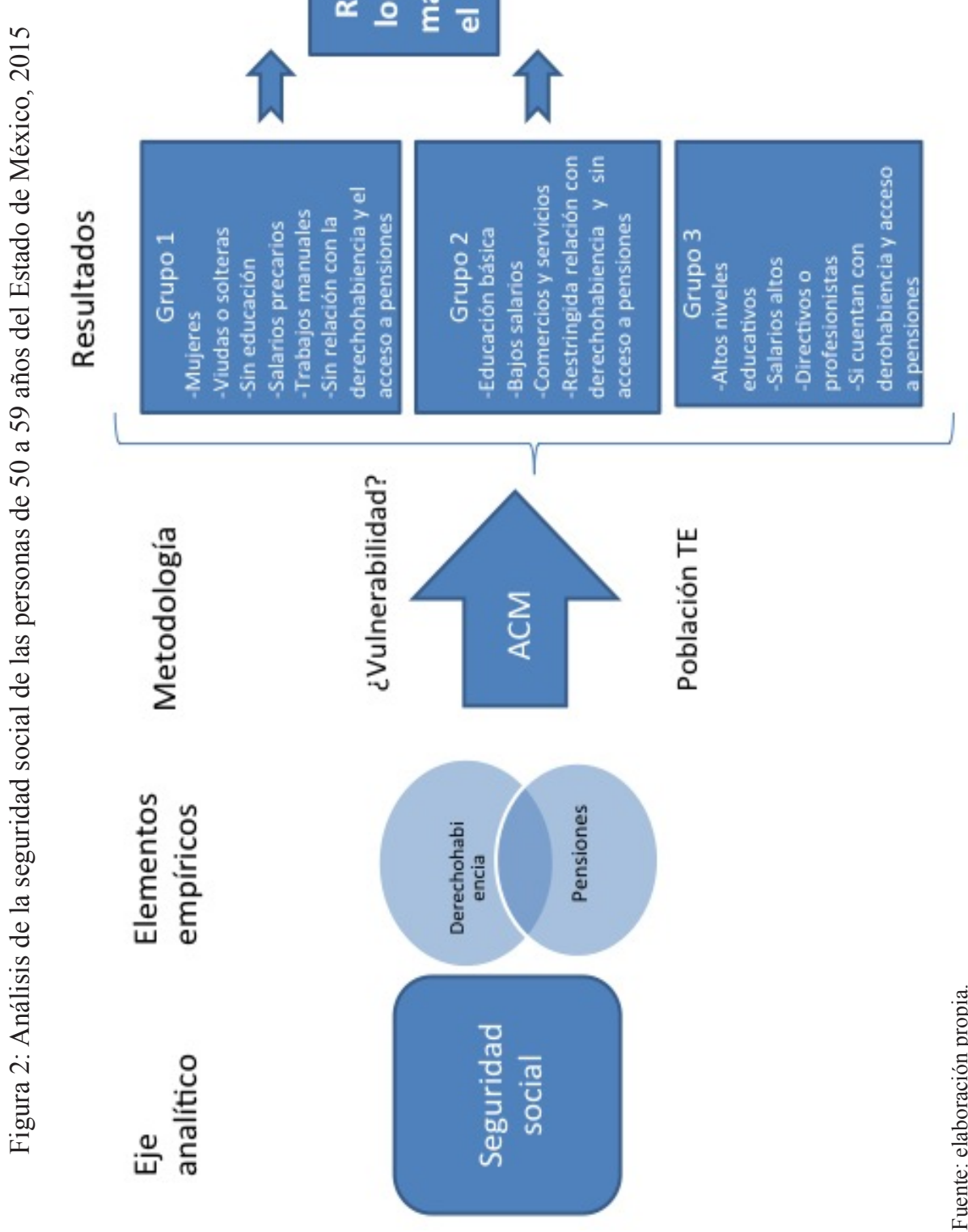


estudio que dificulta su incorporación a la adultez mayor, debido a que la falta de cobertura de seguridad social se suma a rezagos históricos, como falta de capacidad de ahorro, redes sociales y familiares débiles, incertidumbre laboral, entre otras

2. La desigualdad está presente en los trabajadores asalariados de 50 a 59 años del Estado de México, debido a que en el ACM se observan distancias sociales entre los tres grupos analizados, en particular se muestra una polarización asociada con los elementos de la seguridad social.

3. El análisis de la seguridad social en esta investigación expone la heterogeneidad de ésta, mediante tres grupos, asociados con la vulnerabilidad. Es importante señalar que esta heterogeneidad se presenta en una población acotada (grupo de edad y situación en el trabajo), por lo que la presencia de este fenómeno plantea la necesidad de estudiar la falta de cohesión.

4. Por último, esta investigación señala la necesidad de construir un sistema de protección social que proporcione los elementos básicos para las personas en transición hacia el envejecimiento, de tal forma que al incorporarse a la adultez mayor estas personas cuenten con mejores condiciones de seguridad económica y de salud.

\section{REFERENCIAS BIBLIOGRÁFICAS}

Barie, G., 2003, La salud y la derechohabiencia. Instituto Interamericano (III). Comisión Nacional para el Desarrollo de la Población. México.

Bertranou, F., 2005, "Restrictions, problems and dilemmas of social protection in Latin America: Facing the challenges from ageing and income security", in Well-being and Social Policy, vol. 1, núm.1, Second Semester, pp. 33-54.

Bourdieu, P., 2009, Intelectuales, política y poder. Buenos Aires: Eudeba.

Castel, R., 2006, La inseguridad social. ¿Qué es estar protegido? Manantial, Buenos Aires.

CEPAL, 2008, Directrices para la elaboración de módulos sobre envejecimiento en las encuestas de hogares. Centro Latinoamericano y Caribeño de Demografía (CELADE) - División de Población de la CEPAL Naciones Unidas, noviembre, Santiago de Chile.

Díaz, J., 2000, "La seguridad social en México un enfoque histórico (primera parte)", en Revista jurídica de la escuela libre de derecho de Puebla.

Greenacre, M., 1993, Correspondence analysis in practice, Academic Press Limited, USA. 
Guadarrama, R., Hualde A., y López, S., 2012, "Precariedad laboral y heterogeneidad ocupacional: una propuesta teórico-metodológica", en Revista Mexicana de Sociología, 74(2), 213-243.

Haller, W. y Portes, A., 2004, La economía informal, Serie Políticas Sociales, núm. 100, CEPAL.

Ham, R., 1996, "De la solidaridad intergeneracional a la privatización de las pensiones”, en Demos, Carta Demográfica sobre México, IIS-UNAM, México.

INEGI, 2010, XIII Censo de Población y Vivienda 2010, Aguascalientes.

INSP, 2013, Encuesta Nacional de Salud y Nutrición de 2012. Resultados por entidad federativa. Estado de México. Instituto Nacional de Salud Pública (INSP), Cuernavaca.

INSP, 2016, Encuesta Nacional de Salud y Nutrición de Medio Camino (ENSANUT MC 2016). Instituto Nacional de Salud Pública (INSP) Cuernavaca.

López, M., 1987, La Economía del Sistema de Pensiones de la Seguridad Social. Ministerio de Trabajo y Seguridad Social, Compilador, España.

Manrique, B., Salinas, A., Moreno, K., Acosta, I., Sosa, A., Gutiérrez, L., Téllez M., 2013, "Condiciones de salud y estado funcional de los adultos mayores en México”, en Salud Pública de México, vol. 50, núm. 5, pp. S323-S331.

Nugent, R., 1997, La seguridad social: su historia y sus fuentes. En Instituciones de derecho del trabajo y de la seguridad social, Instituto de Investigaciones Jurídicas de la UNAM, pp. 603-622. México.

OIT, 2009, "Responder a la crisis: construir una protección social básica", en $R e$ vista Trabajo, la revista de la Organización Internacional del Trabajo (OIT), núm. 67. Ginebra.

OMS, 2011, Global Health and Aging. Organización Mundial de la Salud (OMS), Génova.

Pedrero, M., 1999, "Situación económica en la tercera edad", en Papeles de población, Centro de Investigación y Estudios Avanzados de la Población/UAEM, Nueva Época año 5, núm. 19, enero-marzo, Toluca.

Rodríguez, J. C., 2003, La economía laboral en el período clásico de la historia del pensamiento económico. Tesis doctoral, Universidad de Valladolid.

Salas, C.,, 1999, "Empleo y tercera edad: dinamismo y tendencias", en Conapo, Envejecimiento demográfico de México: retos y perspectivas, Consejo Nacional de Población, México.

Sánchez, C. A., 2012, "Principales modelos de seguridad social y protección social", en La seguridad social y la protección social en México: su necesaria reorganización, Instituto de Investigaciones Jurídicas de la UNAM. México.

Santín, O., y Alemán, B., 2009, Seguridad Social Sustentable. Biblioteca Mexiquense del Bicentenario. Toluca. 
Shamah, T., Cuevas, L., Mundo, V., Morales, C., Cervantes, L., Villalpando, S., 2008, "Estado de salud y nutrición de los adultos mayores en México: resultados de una encuesta probabilística nacional”, en Salud Pública de México, vol. 50, núm. 5, pp. 383-389.

Vázquez, P., 2013, "Nueva seguridad social y la crisis de las pensiones", en ECONOMÍAunam, 10 (28).

Welti, C., 2013, "Política social y envejecimiento", em Papeles de población, vol 19, núm. 77, Universidad Autónoma del Estado de México, Toluca, pp: 25-59.

\section{RESUMEN CURRICULAR DE LOS AUTORES}

\section{Sergio Cuauhtémoc Gaxiola Robles Linares}

Actuario por la Facultad de Ciencias de la Universidad Nacional Autónoma de México. Maestro en Estudios de Población por la Facultad Latinoamericana de Ciencias Sociales (sede México). Doctor en Estudios de Población por El Colegio de México. Profesor de Tiempo Completo del Centro de Investigación y Estudios Avanzados de la Población de la Universidad Autónoma del Estado de México (UAEM) y miembro del Sistema Nacional de Investigadores (SNI). Docente en programas de licenciatura de la UAEM. Ha publicado varios artículos. Líneas de investigación: envejecimiento, mercados de trabajo y pobreza.

Dirección electrónica: serobles99@gmail.com

\section{Daniel Lozano Keymolen}

He graduated in Sociology from the Universidad Nacional Autónoma de México, Master in Population and Development by FLACSO-México and is a Ph.D. student in Population Studies in Centro de Estudios Demográficos, Urbanos y Ambientales at El Colegio de México A.C. He has participated in the writing of examinations for the accreditation of knowledge for BA in Sociology. He has participated in the book Sociedad, educación y elecciones. Reflexiones desde la Sociología, published by the Universidad Nacional Autónoma de México.

Dirección electrónica: dlozano@colmex.mx

Bernardino Jaciel Montoya Arce

Es Licenciado, Maestro y Doctor en Sociología por la Universidad Nacional Autónoma de México (UNAM). Pertenece al Sistema Nacional de Investigadores Nivel I, cuenta con Perfil Deseable PROMEP. Actualmente 
es Coordinador del Centro de Investigación y Estudios Avanzados de la Población de la Universidad Autónoma del Estado de México (CIEAPUAEM). Entre sus publicaciones recientes se encuentran Demografía indígena en el Estado de México (coautor), 2013; Análisis demográfico del envejecimiento en el Estado de México (compilador), UAEM, 2011 y "La educación indígena en el Estado de México", en Papeles de Población, 2013.

Dirección electrónica:bjmontoyaa@uaemex.mx

\section{Yuliana Gabriela Román Sánchez}

Doctora en Ciencias Económico-Administrativas por la Universidad Autónoma del Estado de México (UAEM). Maestra en Demografía por El Colegio de México, A.C. Licenciada en Actuaría Financiera por la Facultad de Economía de la UAEM. Profesora-Investigadora del Centro de Investigación y Estudios Avanzados de la Población (CIEAP) de la UAEM. Miembro del Sistema Nacional de Investigadores (SNI). Líneas de investigación: envejecimiento demográfico, mercados de trabajo de la población joven y precariedad laboral.

Dirección electrónica: ygromans@uaemex.mx; madon.d126@gmail.com 